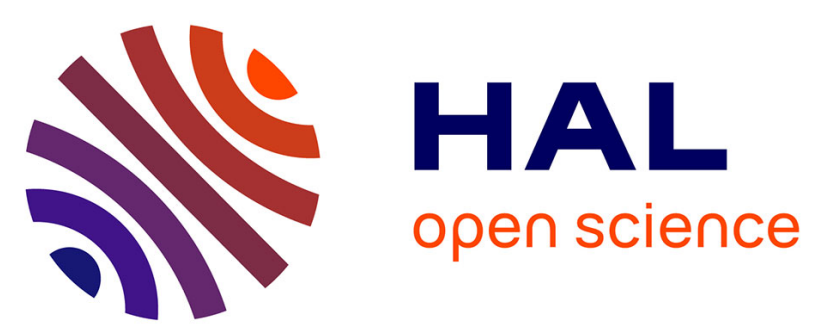

\title{
Femtosecond laser fabrication of micro and nano-disks in single layer graphene using vortex Bessel beams
}

Benjamin Wetzel, Chen Xie, Pierre-Ambroise Lacourt, John Michael Dudley, François Courvoisier

\section{- To cite this version:}

Benjamin Wetzel, Chen Xie, Pierre-Ambroise Lacourt, John Michael Dudley, François Courvoisier. Femtosecond laser fabrication of micro and nano-disks in single layer graphene using vortex Bessel beams. Applied Physics Letters, 2013, 103, pp.241111. 10.1063/1.4846415 . hal-00924224

\section{HAL Id: hal-00924224 \\ https://hal.science/hal-00924224}

Submitted on 6 Jan 2014

HAL is a multi-disciplinary open access archive for the deposit and dissemination of scientific research documents, whether they are published or not. The documents may come from teaching and research institutions in France or abroad, or from public or private research centers.
L'archive ouverte pluridisciplinaire $\mathbf{H A L}$, est destinée au dépôt et à la diffusion de documents scientifiques de niveau recherche, publiés ou non, émanant des établissements d'enseignement et de recherche français ou étrangers, des laboratoires publics ou privés. 


\title{
Femtosecond laser fabrication of micro and nano-disks in single layer graphene using vortex Bessel beams
}

\author{
Benjamin Wetzel a) Chen Xie, Pierre-Ambroise Lacourt, John M. Dudley, and Francois Courvoisier \\ Département d'Optique P.M. Duffieux, Institut FEMTO-ST, UMR-6174 CNRS, \\ Université de Franche-Comté, 25030 Besançon, France
}

\begin{abstract}
We report the fabrication of micro and nano-disks in single layer chemical vapor deposition graphene on glass substrate using femtosecond laser ablation with vortex Bessel beams. The fabricated graphene disks with diameters ranging from $650 \mathrm{~nm}$ to $4 \mu \mathrm{m}$ were characterized by spatially resolved micro-Raman spectroscopy. The variation of ablation threshold was investigated as a function of the number of pulses showing an incubation effect. A very high degree of size control of the fabricated graphene disks is enabled using a sequence of femtosecond pulses with different vortex orders.
\end{abstract}

Since its original synthesis in $2004, \frac{1}{1}$ graphene has been intensely studied due to its potential dramatic impact in many areas of science and technology 23$]$ There has been particular recent interest in creating patterned or structured graphene on the micro and nanoscales, and applications such as complete optical absorption 4 and $\mathrm{THz}$ antennas ${ }^{5}$ have been recently obtained using structured graphene ribbons, disks and rings. $\underline{6}$

Current processes for graphene patterning and structuring use well-known clean-room technologies such as e-beam lithography, wet-chemical etching or focused ion beam (FIB) milling. ${ }^{9}$ However, it has also been shown that femtosecond laser ablation is also a highly flexible technique to fabricate graphene structures, with particular advantage in rapid prototyping. Moreover, the highly material-dependent threshold for femtosecond laser ablation allows patterning of graphene structures on arbitrary substrates 10 , and controlled ablation layer per layer.1112 Previous studies of ultrafast laser ablation of graphene have shown that pristine and chemical vapor deposition (CVD) graphene have well defined ablation thresholds over which clean ablation is performed with no residues whereas, below this threshold, defects are formed and serve as initiating centers for ablation by consecutive pulses ${ }^{13}$ Using this cumulative effect, a range of structures including micro- and nano-ribbons and nanodots have been processed in graphene using femtosecond lasers in the $\mathrm{kHz}$ and $\mathrm{MHz}$ regimes. $12 \mid 16$

Although the threshold effect in the single shot regime can be used to generate nanostructures of dimension well below the laser wavelength, the precise positioning of the sample with respect to the laser beam waist is an extremely severe constraint. 17 The use of nondiffracting Bessel beams, however, drastically reduces this constraint since their shape is invariant over a longitudinal distance much longer that the Rayleigh range of equivalent Gaussian beams. The use of Bessel beams in fabricating a wide range of structures in dielectric media is well known ${ }^{18}$ but, in this Letter, we report the application of femtosecond non-diffracting Bessel beams in

a) Electronic mail: benjamin.wetzel@femto-st.fr structuring CVD graphene on the micron and nanometer scale. For the important applications in creating ringlike and disk-like structures in graphene, we use spatially shaped higher-order vortex Bessel beams (VBB), that consist of a series of concentric annular cylinders surrounding a central zone of zero intensity. The use of VBB for ablation allows the fabrication of isolated graphene disks surrounded by concentric rings by direct ablation of the targeted shape, even using single laser shots, thus greatly reducing both the processing time and constraint on sample positioning compared to machining with Gaussian beams. The morphology of the fabricated structures is characterized by Scanning Electron Microscopy (SEM), confocal microscopy and micro-Raman spectroscopy 19$] 21$ Studies of the intensity threshold dependence of the ablation process in the single and multishot regime allow us to show how a carefully chosen sequence of Bessel pulses with different vortex orders allows removal of the surrounding ring structures and the production of highly isolated micro- and nano-disks.
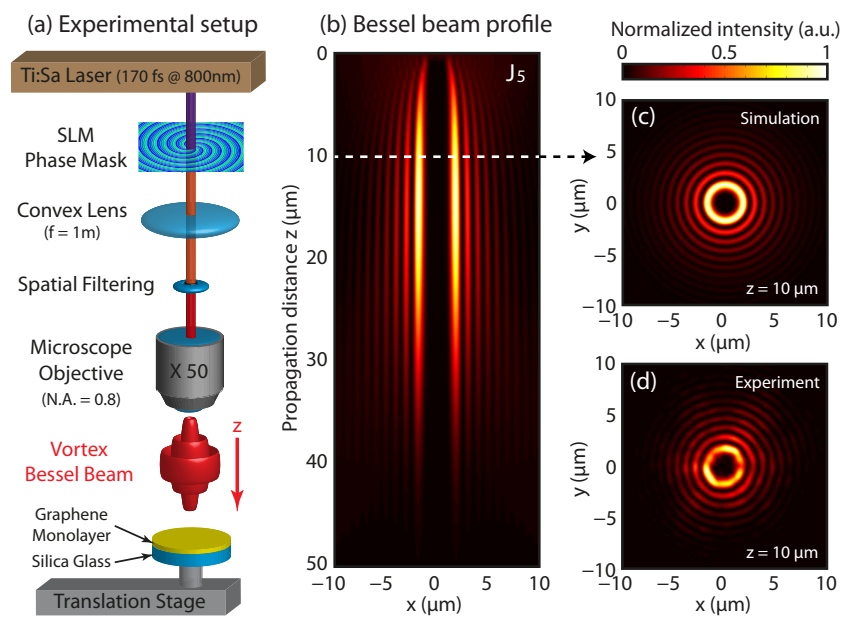

FIG. 1. (a) Experimental setup of vortex Bessel beams graphene structuring. Demagnification of imaging system is $1 / 278$. (b) Longitudinal intensity map of $J_{5}$ Bessel beam obtained numerically. (c) Numerical and (d) experimental transverse intensity maps of $J_{5}$ at $z=10 \mu \mathrm{m}$. 


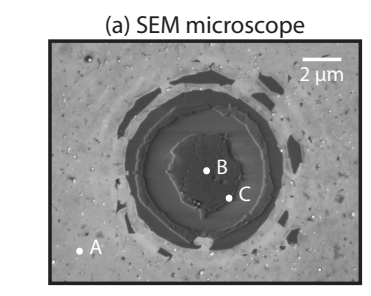

(c) Raman spectra
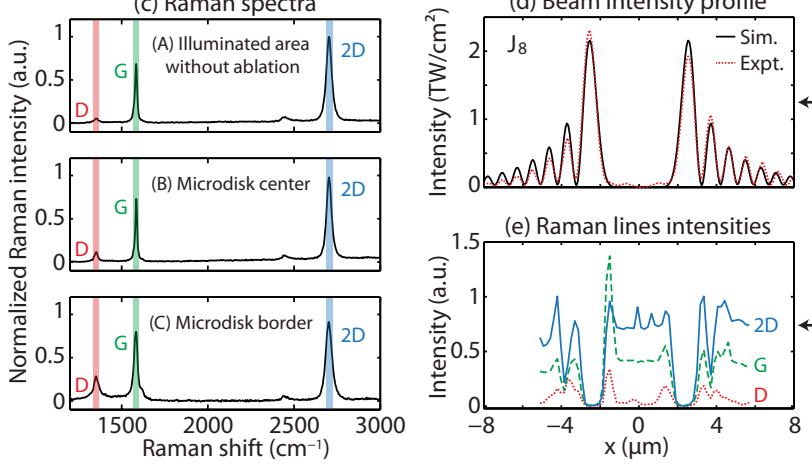

(e) Raman lines intensities

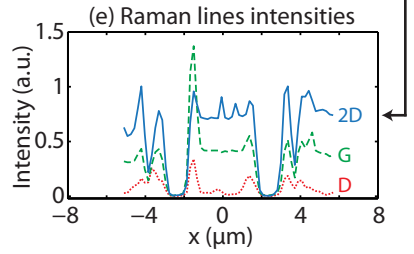

FIG. 2. Single-shot ablation by a $J_{8}$ beam : (a) SEM and (b) optical confocal reflectivity images of structured graphene. (c) $\mu$-Raman spectra obtained at three sample locations shown in (a). (d) Numerical and experimental beam transverse profile. (e) Evolution of Raman spectral peak intensities (respectively D, G and 2D), as highlighted in (c), along the slice of the structure shown as a dashed line.

Fig. 1(a) shows the experimental setup. We use a Ti:Sapphire regeneratively-amplified laser system delivering $170 \mathrm{fs}$ pulses at $800 \mathrm{~nm}$ with beam shaping provided from a spatial light modulator (SLM). The laser can operate at $\mathrm{kHz}$ repetition rates and pulse-picking can be implemented to allow single-shot operation. The applied spatial phase has the form $\phi_{n}(r, \theta)=2 \pi r / \rho_{0}+n \theta$ where the first term generates a Bessel beam and the second imposes vorticity of order $n$ that determines the central ring radius. After demagnification and spatial filtering within a $4 \mathrm{f}$ telescope, ${ }^{22}$ we obtain a vortex Bessel beam $J_{n}, \stackrel{23 \mid 24}{2}$ which is then incident on a commercial monolayer graphene CVD sample on a glass substrate. The sample position is controlled with sub-micron precision. Fig. 1(b) shows the expected (calculated) longitudinal intensity distribution of a typical VBB of order 5. Figs. 1(c) and $1(\mathrm{~d})$ present respectively calculated and experimental cross sections at a propagation distance of $z=10 \mu \mathrm{m}$ (from the focus of the microscope objective) where variation of the intensity with distance is small. This region is where we positioned samples in our experiments.

The first ablation results we present are in Fig. 2, where our graphene sample was illuminated with a single $J_{8}$ pulse of energy $E=150$ nJ. Fig. 2(a) shows an SEM image of the resulting structure where we see a central $4 \mu \mathrm{m}$ diameter microdisk of unablated graphene surrounded by several ablated concentric rings. The first ring has a typical width of $500 \mathrm{~nm}$. When interpreting the SEM image, note that the graphene regions inside the ring structure are electrically isolated from the surrounding graphene film and thus appear visually different. The same sample was imaged with confocal microscopy in reflection configuration, showing the same reflectivity for the central microdisk and the remaining film. Numerous scattering centers are visible in this image but such scattering can in fact be observed in CVD graphene samples prior to any ablation and are attributed to residues from the CVD process. We note from Fig. 2(b) higher reflectivity at the disk and ring edges, attributed to folding of a part of the ablated graphene layer and possible debris redeposition. From Figs. 2(a) and 2(b), small nonuniformity in the ablation structure is visible, mainly due to a non-symmetric power distribution in the experimental beam, although as shown in Fig. 2(d), the measured experimental beam radial profile is very close to an ideal calculated profile of the $J_{8}$ vortex beam.

The sample was further characterized by micro-Raman spectroscopy 20 with laser excitation at $473 \mathrm{~nm}$ and $400 \mathrm{~nm}$ spatial resolution. In Fig. 2(c), we compare the Raman spectra acquired at different points on the sample as shown in Fig. 2(a): (A) outside the ablated structure, (B) in the center of the microdisk and $(\mathrm{C})$ on its edge. The spectra measured at (A) and (B) are almost identical showing that the graphene structure is almost unaffected during ablation process. The slightly elevated increase of the defect D-band in (B) and (C) (and the broader Gband in $(\mathrm{C})$ ) indicates higher disorder in the lattice structure that originates both from edge effects ${ }^{19 \mid 21}$ and folding in accordance with observations by other groups. 13115

In Fig. 2(e), we plot the Raman spectra peak intensities 19 21 (respectively D, G and 2D-bands as highlighted in Fig. 2(c) shaded areas) along a line of the ablated microdisk as indicated by the dotted line in Fig. 2(b). We observe a contrast exceeding 50:1 in the Raman signal between the non-ablated and ablated regions attesting that graphene has been completely removed. The enhancement of disorder (D-band) at structure edges is also apparent. Comparing these Raman intensities with the corresponding beam radial intensity presented in Fig. 2(c), we can see a clear correspondence between the graphene ablated regions and the lobes of the $J_{8}$ beam highlighting a threshold-like behavior in the single-shot femtosecond laser ablation process.

To further investigate graphene femtosecond laser structuring, we measured the ablation threshold of our CVD graphene samples using a beam with vortex order $n=5$. Taking advantages of the characterized VBB intensity profile, ${ }^{23}$ it is straightforward to determine the ablation threshold of CVD graphene associated with complete removal of the material detected from confocal reflectivity images. These results are shown in Fig. 3 where we plot the intensity threshold depending on the number of individual pulse exposures. Each ablation threshold value was obtained by performing a statistical analysis on 60 measurements. Calculated error bars include the influence of beam and sample inhomogeneities as well as laser power fluctuations (separately 




FIG. 3. Intensity ablation thresholds (CVD monolayer graphene, 170 fs pulses) measured experimentally depending on the number of exposures $\mathrm{N}$.

measured $<5 \%$ RMS). We find that, for single-shot exposure $(N=1)$, single layer graphene was removed above an intensity of $860 \pm 180 \mathrm{GW} \mathrm{cm}^{-2}$, corresponding to a fluence of $156 \pm 33 \mathrm{~mJ} \mathrm{~cm}^{-2}$. These results are in good agreement with previously reported single-shot ablation thresholds ranging from $150-210 \mathrm{~mJ} \mathrm{~cm}^{-2}$ for slightly different types of graphene samples and pulse durations. ${ }^{13 / 14 \mid 25}$ Interestingly, as the number of laser shots $N$ is increased, we observe a drastic reduction of the ablation threshold arising from a cumulative effect in graphene ablation process ${ }^{14}$ also suggesting a saturationlike behavior of the ablation threshold observed for a high number of exposures (i.e. $N>100$ ).

We emphasize that, with the single-shot $J_{8}$ beam configuration presented in Fig. 2, a $10 \%$ fluctuation in the incident pulse energy or, equivalently, a sample positioning change of $2.3 \mu \mathrm{m}$, will induce a variation in the microdisk radius of only $27 \mathrm{~nm}$ (corresponding to an error below $1.5 \%$ ). This arises from both the high steepness of the main ring intensity profile and the nondiffracting behavior of VBB. The same sample positioning error using a gaussian beam with similar width as the main ring of the VBB, would change the ablation radius by $250 \mathrm{~nm}$, i.e. one order of magnitude higher than using a VBB. Importantly, a similar comparison holds between VBB and beams resulting from focal spot shaping 26 that rapidly diffract before and after the focus. This further highlights the advantage of using diffraction-free Bessel beams for high-resolution high-speed machining.

We can apply these results to generate an isolated microdisk using multiple shot exposures of the sample with a sequence of VBB specifically chosen to remove the surrounding graphene rings observed in Fig. 2. Specifically, the sample was illuminated by 4 pulses of same energy $(E=200 \mathrm{~nJ})$ but with vorticity order $n$ of the Bessel beam increased between subsequent exposures $(n=8,9,10,11)$. As shown in Figs. 4(a)-4(c), this sequence defines a doughnut-shaped region where graphene was completely removed over a diameter of $11 \mu \mathrm{m}$ while preserving a microdisk of $3.9 \mu \mathrm{m}$ diameter at the center, in good agreement with the shape expected from the ablation threshold indicated as dashed lines. We can see
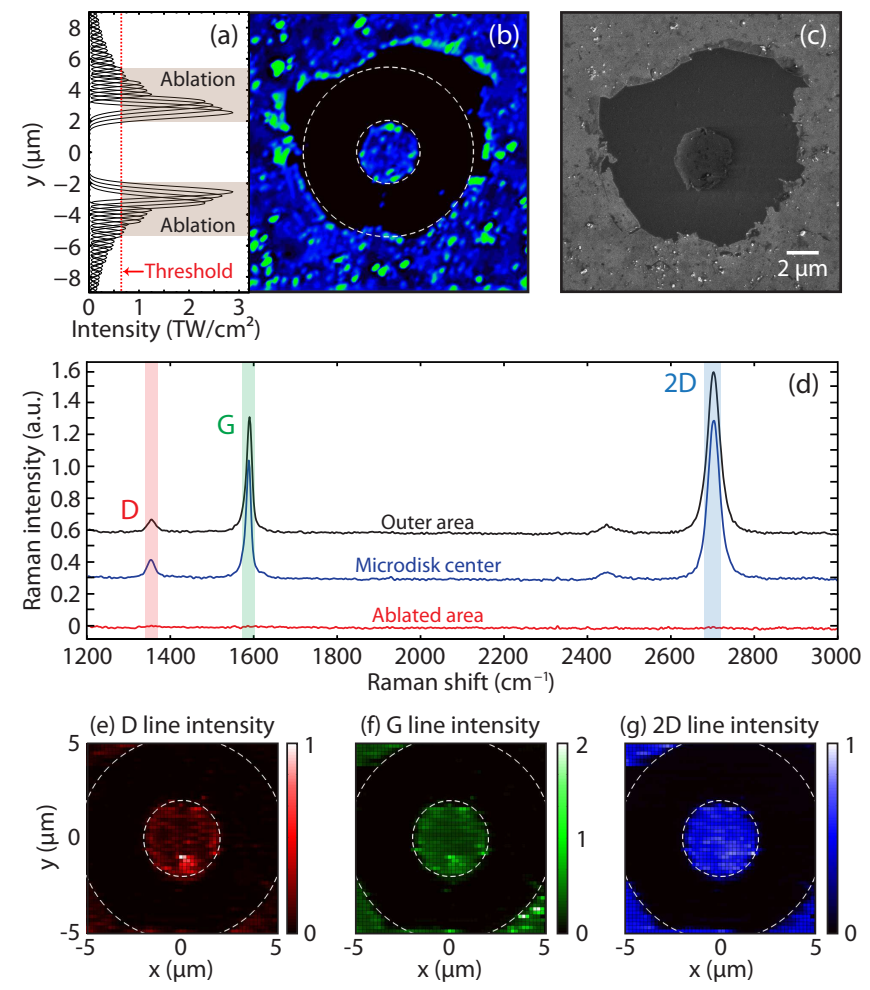

FIG. 4. (a) Transverse profile of 4 superimposed Bessel beams intensities $\left(J_{8}, J_{9}, J_{10}, J_{11}\right)$ and expected ablation profile (shaded area) for intensities above ablation threshold (red dashed line - for 4 laser expositions). (b) Confocal microscopy and (c) SEM images of the exposed sample. (d) Raman spectra at various locations in the sample. (e)-(g) Corresponding Raman maps of graphene peaks intensities. Expected ablation limits are reported with white dashed circles.

that the microdisk is free from fold and exhibits better shape uniformity (i.e. edge quality) than the disk shown in Fig. 2.

Fig. 4(d) shows the Raman spectra measured in the disk center, in the ablated area and outside the ablation zone, confirming that even with 4 exposures, the graphene structure in the disk remains preserved. In addition, we realized spatial mappings of each Raman spectral bands D, G and 2D, presented in Figs. 4(e)$4(\mathrm{~g})$, where one can see quasi-uniform peak resonance intensities in the remaining graphene area. Such constant intensity maps of Raman peaks in the disk region (except where ablated nanoparticles were redeposited) confirms the overall quality of our Bessel femtosecond ablation technique. In addition, we can see a slight increase in the D-band intensity in the disk compared to the remaining graphene layer outside the ablated region that is associated with edge effects.

The structural quality enhancement obtained with multiple exposures was confirmed by an analysis of a data set over a large range of parameters including vorticity. We attribute the increase of edge-quality with 
(a) Microdisk (3.6 $\mu \mathrm{m}$ diameter)
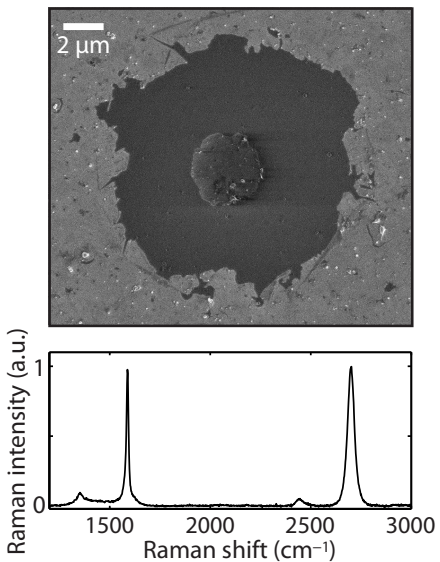

(b) Nanodisk (650 $\mathrm{nm}$ diameter)
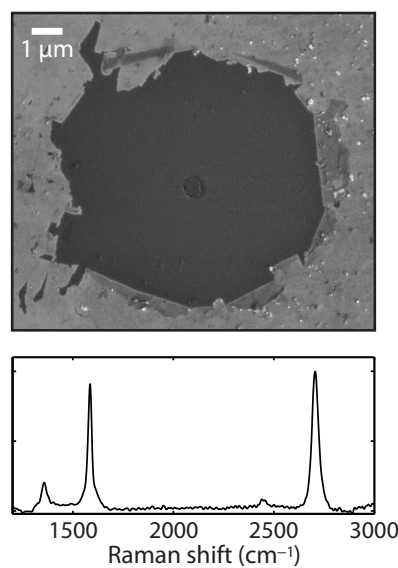

FIG. 5. (Top) SEM images and (Bottom) normalized Raman spectra measured at the structure center for isolated graphene (a) microdisk and (b) nanodisk obtained from ablation by 20 superimposed vortex Bessel beam exposures.

subsequent illuminations to preferential ablation of the folds and defects on the edges from reduction of the ablation threshold. In the following, we take advantage of this observation and perform further experiments using a greater number of laser shots. Fig. 5 shows images of isolated micro and nano-disks processed by 5 exposures of 4 different beams with increasing vorticity $n$ so that the overall number of shots is 20 . In Fig. 5(a), we used a sequence of shots as follows: $5 J_{8}, 5 J_{9}, 5 J_{10}$ and $5 J_{11}$ with $200 \mathrm{~nJ} /$ shot. We see in the figure an isolated graphene microdisk with an overall better symmetry and a slightly reduced diameter $(3.6 \mu \mathrm{m})$ due to the reduced ablation threshold arising from a higher number of pulse exposures. In Fig. 5(b), we decreased the vortex orders of the Bessel beams and used the sequence $5 J_{2}, 5 J_{3}, 5 J_{4}$ and $5 J_{5}$ at $80 \mathrm{~nJ} /$ shot so that we obtained a nanoscale disk with an average diameter of $650 \mathrm{~nm}$ which is, as expected, highly isolated from the surrounding graphene film. In this case, the ablation radius is more than 5 times larger than the nanodisk radius itself. Raman analysis was also performed at the center on each disk as presented in Fig. 5 and interestingly shows a good structural quality even for the nanodisk.

In conclusion, we have investigated femtosecond laser ablation of CVD monolayer graphene by vortex Bessel beams under single and multiple pulse illumination. Intensity thresholds for ablation have been determined and the ablation sites have been carefully characterized by micro-Raman spectroscopy. The crystalline structure of graphene is unaffected from a few hundreds of nanometers away from the ablation edge. The non-diffracting behavior of vortex Bessel beams is an important advantage to avoid non-critical sample positioning and therefore allow for fast patterning over large non-flat surfaces. Micro-disks surrounded by nano-rings were obtained in the single-shot regime and the successive exposures using several vortex beams with different topological charges generates isolated graphene microdisks and nanodisks down to $650 \mathrm{~nm}$ in diameter. This work opens up alternative routes for laser micro-nano structuring of graphene and we anticipate widespread applications of this approach for fast laser processing in 2D materials.

This work was supported by the Région of FrancheComté and the French ANR, contract 2011-BS04-010-01 NANOFLAM. This work has been performed in cooperation with the Labex ACTION program (contract ANR-11-LABX-01-01). The authors thank M. Moreau and CNRS, UMR 8516 LASIR, Univ. Lille 1 (France) for micro-Raman spectroscopy characterization as well as D. Charraut and F. Palmino for sample imaging.

${ }^{1}$ K. S. Novoselov, A. K. Geim, S. V. Morozov, D. Jiang, Y. Zhang, S. Dubonos, I. V. Grigorieva, and A. A. Firsov, Science 306, 666 (2004).

${ }^{2}$ A. K. Geim, Science 324, 1530 (2009).

${ }^{3}$ F. Bonaccorso, Z. Sun, T. Hasan, and A. C. Ferrari, Nature Photon. 4, 611 (2010).

${ }^{4}$ S. Thongrattanasiri, F. H. L. Koppens, and F. J. G. de Abajo, Phys. Rev. Lett. 108, 047401 (2012).

${ }^{5}$ P. Liu, W. Cai, L. Wang, X. Zhang, and J. Xu, Appl. Phys. Lett. 100, 153111 (2012).

${ }^{6}$ L. Ju, B. Geng, J. Horng, C. Girit, M. Martin, Z. Hao, H. A. Bechtel, X. Liang, A. Zettl, Y. R. Shen, and F. Wang, Nature Nanotech. 6, 630 (2011).

${ }^{7}$ W. Wang, J. Phys.: Condens. Matter 24, 402202 (2012).

${ }^{8}$ M. Hashemi, M. H. Farzad, N. A. Mortensen, and S. Xiao, J. Opt. 15, 055003 (2013).

${ }^{9}$ J.-Y. Hong and J. Jang, J. Mater. Chem. 22, 8179 (2012).

${ }^{10}$ J. B. Park, J.-H. Yoo, and C. P. Grigoropoulos, Appl. Phys. Lett. 101, 043110 (2012).

${ }^{11}$ S. Dhar, A. R. Barman, G. X. Ni, X. Wang, X. F. Xu, Y. Zheng, S. Tripathy, Ariando, A. Rusydi, K. P. Loh, M. Rubhausen, A. H. C. Neto, B. Ozyilmaz, and T. Venkatesan, AIP Advances 1, 022109 (2011).

${ }^{12}$ R. J. Stohr, R. Kolesov, K. Xia, and J. Wrachtrup, ACS Nano 5, 5141 (2011).

${ }^{13}$ A. Roberts, D. Cormode, C. Reynolds, T. Newhouse-Illige, B. J. LeRoy, and A. S. Sandhu, Appl. Phys. Lett. 99, 051912 (2011).

${ }^{14}$ M. Currie, J. D. Caldwell, F. J. Bezares, J. Robinson, T. Anderson, H. Chun, and M. Tadjer, Appl. Phys. Lett. 99, 211909 (2011).

${ }^{15}$ J.-H. Yoo, J. Bin In, J. Bok Park, H. Jeon, and C. P. Grigoropoulos, Appl. Phys. Lett. 100, 233124 (2012).

${ }^{16}$ G. Kalita, L. Qi, Y. Namba, K. Wakita, and M. Umeno, Mater. Lett. 65, 1569 (2011).

${ }^{17}$ A. P. Joglekar, H.-H. Liu, E. Meyhöfer, G. Mourou, and A. J. Hunt, Proc. Natl. Acad. Sci. U. S. A. 101, 5856 (2004).

${ }^{18}$ M. K. Bhuyan, F. Courvoisier, P.-A. Lacourt, M. Jacquot, R. Salut, L. Furfaro, and J. M. Dudley, Appl. Phys. Lett. 97, 081102 (2010).

${ }^{19}$ A. C. Ferrari, J. C. Meyer, V. Scardaci, C. Casiraghi, M. Lazzeri, F. Mauri, S. Piscanec, D. Jiang, K. Novoselov, S. Roth, and A. K. Geim, Phys. Rev. Lett. 97, 187401 (2006).

${ }^{20}$ D. Graf, F. Molitor, K. Ensslin, C. Stampfer, A. Jungen, C. Hierold, and L. Wirtz, Nano Lett. 7, 238 (2007).

${ }^{21}$ L. M. Malard, M. A. Pimenta, G. Dresselhaus, and M. S. Dresselhaus, Phys. Rep. 473, 51 (2009).

${ }^{22}$ F. Courvoisier, P.-A. Lacourt, M. Jacquot, M. K. Bhuyan, L. Furfaro, and J. M. Dudley, Opt. Lett. 34, 3163 (2009). 
${ }^{23}$ V. Jarutis, R. Paškauskas, and A. Stabinis, Opt. Commun. 184, 105 (2000).

${ }^{24}$ J. Arlt and K. Dholakia, Opt. Commun. 177, 297 (2000).

${ }^{25}$ W. Zhang, L. Li, Z. B. Wang, A. A. Pena, D. J. Whitehead, M. L. Zhong, Z. Lin, and H. W. Zhu, Appl. Phys. A: Mater. Sci.
Process. 109, 291 (2012).

${ }^{26}$ N. Sanner, N. Huot, E. Audouard, C. Larat, J.-P. Huignard, and B. Loiseaux, Opt. Lett. 30, 1479 (2005). 